\title{
Assessing scarlet fever re-emergence from notifiable disease surveillance in Hong Kong
}

\author{
Chun Fan Lee, Benjamin J. Cowling and Eric H. Lau*
}

School of Public Health, The University of Hong Kong, Hong Kong, China

\section{Objective}

This study examined the epidemiology of scarlet fever in Hong Kong based on notifiable disease surveillance data, in a period where a 10-fold upsurge in scarlet fever incidence occurred. High risk groups and important factors associated with scarlet fever transmission were identified.

\section{Introduction}

Scarlet fever is a notifiable disease in Hong Kong for over 40 years. There was relatively low activity of scarlet fever until an outbreak in mid-2011 which resulted in two deaths and more than 1,500 cases. Scarlet fever incidence remained elevated since then with $>10$-fold increase comparing to that before the upsurge $(1,2)$. Reemergence of scarlet fever was also reported in China in 2011 and the United Kingdom in 2014 (3). We analyzed the patterns in scarlet fever incidence in Hong Kong using the notifiable disease surveillance data from 2005-2015.

\section{Methods}

We analyzed 7,266 scarlet fever cases aged 14y or younger from 2005-2015, who were notified to the Department of Health. Hierarchical multivariable negative binomial models were fitted to the data to study the effects of age, sex, school holidays, and other meteorological parameters, accounting for autocorrelation, seasonal and long-term trend. Separate models were fitted to the data before and after the upsurge in 2011, excluding data in 2011 to allow for a 1 -year window period.

\section{Results}

We observed seasonal pattern throughout the study period (Figure). Among children aged $\leq 5 \mathrm{y}$, the average scarlet fever incidence was 3.3 per 10,000 children in $2005-2010$, which increased substantially to 18.1 per 10,000 children in 2012-2015.

The final model included age, sex, school holidays in the preceding week, temperature, relative humidity, rainfall, long-term and bimodal seasonal trend. Based on the model, we identified no significant longterm trend before the upsurge in 2011, but there was a mild decreasing trend of about $8 \%(95 \% \mathrm{CI}=6-11 \%)$ per year after the upsurge. A major peak was identified in December to January, with a milder peak in May to June.

We found that the most affected groups were kindergarten students (3-5y), followed by primary school students $(6-11 y)$. Comparing to girls aged $0-2 y$, boys had significantly higher risk than girls except for the $0-2 y$ age group, and boys aged $3-5 y$ had the highest risk (adjusted incidence rate ratio $(\mathrm{IRR})=1.47,95 \% \mathrm{CI}=1.32-1.65)$. School holidays were significantly associated with lower incidence of scarlet fever, with an adjusted IRR of $0.58(95 \% \mathrm{CI}=0.51-0.65)$ after the upsurge in 2011. Temperature was found to be negatively associated with scarlet fever incidence (adjusted IRR $=0.963,95 \% \mathrm{CI}=0.940-0.987$ ) after the upsurge.

\section{Conclusions}

Our study showed that elevated activity of scarlet fever was sustained for more than 5 years after the upsurge in 2011. We found that younger children who started schools, especially for boys aged
3-5 years, had a higher risk of scarlet fever, and there was significant effect of school holidays in reducing scarlet fever incidence. Combining these findings, school-based control strategy is likely to be effective. Sustained and consistent surveillance of scarlet fever allows continued monitoring of potential change in high risk group to drive updated and effective control strategy.
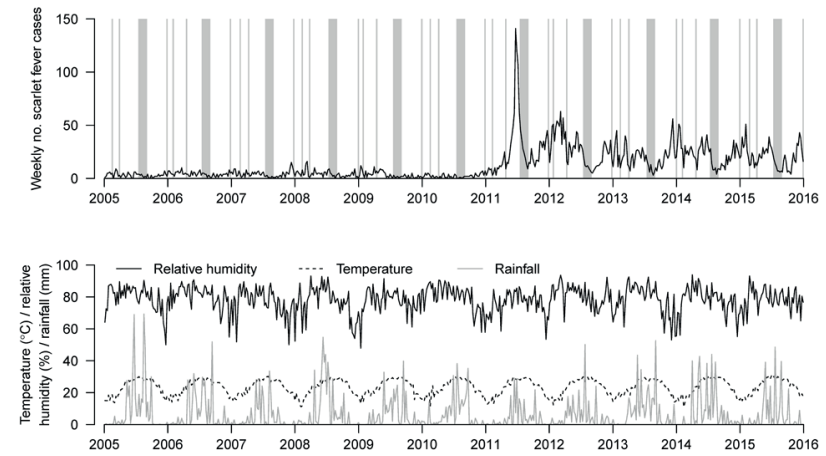

Weekly number of notified scarlet fever cases, Hong Kong, 2005-2015. Gray bars indicate periods of school holidays (top). Weekly average of temperature, relative humidity, and rainfall (bottom).

\section{Keywords}

scarlet fever; re-emergence; children; school holiday

\section{Acknowledgments}

We thank the Centre for Health Protection, Department of Health, in Hong Kong for their kind support and assistance in collating the notification data. This work is supported by the Health and Medical Research Fund of the Food and Health Bureau (grant no. HKS-16-E09) and the Harvard Center for Communicable Disease Dynamics from the National Institute (grant no. U54 GM088558). The content is solely the responsibility of the authors and does not necessarily represent the official views of the National Institute of General Medical Sciences or the National Institutes of Health.

\section{References}

1. Lau EH, Nishiura H, Cowling BJ, Ip DKM, Wu JT. Scarlet fever outbreak, Hong Kong, 2011. Emerging Infectious Diseases. 2012 Oct;18(10):1700-2.

2. Centre for Health Protection. Number of notifiable infectious diseases by month in 2011. http://www.chp.gov.hk/en/data/1/10/26/43/455. $\mathrm{html}$

3. Lee CF, Cowling BJ, Lau EHY. Epidemiology of Reemerging Scarlet Fever, Hong Kong, 2005-2015. Emerg Infect Dis. 2017 Oct;23(10):1707-1710.

*Eric H. Lau

E-mail: ehylau@hku.hk 